\title{
Processing and Use of Satellite Images in Order to Extract Useful Information in Precision Agriculture
}

\author{
Mihai Valentin HERBEI ${ }^{*}$, Cosmin Alin POPESCU ${ }^{2}$, Radu BERTICI ${ }^{3}$, Adrian SMULEAC ${ }^{4}$, George POPESCU \\ ${ }^{1}$ Cartography and GIS, Banat University of Agricultural Sciences and Veterinary Medicine, "Regele \\ Mihai I al României", Timisoara, Romania \\ ${ }^{2}$ Remote sensing and Cadaster, Banat University of Agricultural Sciences and Veterinary Medicine, \\ "Regele Mihai I al României", Timisoara, Romania \\ ${ }^{3}$ Soil Science, Banat University of Agricultural Sciences and Veterinary Medicine "Regele Mihai I al \\ României" from Timisoara, Romania \\ ${ }^{4}$ Topography and GNSS, Banat University of Agricultural Sciences and Veterinary Medicine "Regele \\ Mihai I al României" from Timisoara, Romania \\ ${ }^{5}$ Cadaster, Banat University of Agricultural Sciences and Veterinary Medicine, "Regele Mihai I al \\ României", Timisoara, Romania \\ * corresponding author, e-mail: mihai_herbei@yahoo.com
}

Bulletin UASVM series Agriculture 73(2)/2016

Print ISSN 1843-5246; Electronic ISSN 1843-5386

DOI 10.15835/buasvmcn-agr: 12442

\begin{abstract}
Image analysis methods were developed and diversified greatly in recent years due to increasing speed and accuracy in providing information regarding land cover and vegetation in urban areas. The aim of this paper is to process satellite images for monitoring agricultural areas. Satellite images used in this study are medium and high resolution images taken from QuickBird and SPOT systems. Based on these images, a supervised classification was performed of a very large area, having as result the land use classes. Supervised classification can be defined as the ability to group the pixels that compose the satellite image, digitally, in accordance with their real significance. Gaussian algorithm of maximum similarity (Maximum likelihood) was used, referred to in the specialty literature as maximum likelihood method or probabilistic classification, and based on the use of probability theory (function Gaussian) to compare the spectral values of each pixel in hand with statistical " fingerprint "of each area of interest. Practically, conditional probabilities were calculated of belonging to one class or another. The points in the middle of the group have a higher probability of belonging to the certain class, probability intervals (concentric isolines or contours of equal probability) being delimited graphically by izocontours expressing spectral variations within each set of training.
\end{abstract}

Keywords: classification, remote sensing, resolution.

\section{INTRODUCTION}

The usefulness of remote sensing in agriculture has been observed for a long time, especially by researchers originating from the United States of America, which began to introduce remote sensing in their studies with the purpose of implementing of a more efficient agriculture. The first studies began even before the development of computerized remote sensing, agronomists noticing the advantages of using satellite and photogrammetric images in order to develop agriculture in the 70s. The first such studies have focused on estimating agricultural production using photogrammetric and satellite imagery (Bauer 1975) (Idso, Jackson and Reginato 1977), followed by studies on identifying areas with the irrigation necessity (Allan 1983) and even studies on the spatial distribution of crops and crop types 
(Wall, Thomas și Brown 1984 Herbei et al., 2015). Studies on the use of remote sensing for agriculture are focused on eight aspects: identifying the health of plants, identifying the need for irrigation, identifying areas with weeds, trying to predict drought, trying to estimate the production of crops, identifying the main types of crops, but also studies on vineyards or orchards. The methods of image analysis were developed and diversified greatly in recent years due to increasing speed and accuracy in providing information on land cover and vegetation in urban areas Classification of satellite imagery, although it is an older analysis method, it continuously improved work precision, as a result of high-resolution images provided by the new generation of satellites, as well as more effective algorithms and more efficient methods of analysis (Huang and colab.2009). Methods based on the analysis of satellite images allow evolutionary analysis of vegetation cover and crop structured with high accuracy (Meliadis and Meliadis 2011). Changes related to the using/ covering the lands represent an important part of global changes affecting the environment (Begov Ungur A. 2015; Begov Ungur A. 2013; Smuleac L. et al. 2013). These changes appear by altering (increasing or decreasing) the density and composition of land details (Horablaga et al. 2013) as well as the conditions, all registered on the satellite image (Rogan et al. 2003). Mapping and monitoring changes related to the use/cover of the lands are essential operations to understanding the mechanism underlying the changes and modeling their impact, at different levels, over the environment and ecosystems (William et al. 1994). Approaches related to the identification of areas with changes / without changes in the use / cover of the land are considering to modify some attributes of the landscape (Horablaga et al. 2012) which can be measured continuously (Coppin et al. 2001; Rogan and Chen 2004). All these changes can be identified, measured and monitored using methods specific to satellite remote sensing. Depending on how the changes are identified, there are two categories of methods (Lu et al.2004): methods that identify changes in the form of binary maps which show surfaces with / without changes, such as the difference between images, the difference between vegetation indices, etc., and methods that highlight in detail the trajectory of the changes "from - to" such as post- classification comparison and hybrid methods of identifying changes. Considering the ability of locating changes, the specialty literature classifies the methods in post-classification and preclassification (Yuan et al. 1998). Pre-classification methods can locate the changes but they do not show their nature (Singh 1989; Yuan et al. 1998) while post- classification methods allow the localization of the changes, as well as their nature. The literature is analyzing a wide variety ofmethods to identify the changes (Yuan et al. 1998; Lu et al. 2004). The basic principles for satellite image classification is based on analysis and placement of pixels with similar characteristics of brightness in the same group. The two types of classification, unsupervised and supervised (groups of classes defined by the operator) ensure a different accuracy in the analysis of satellite images (Akgün et al., 2004). In supervised classification there are several methods with different accuracies: the minimum distance (73.77\%), parallelepiped method (34.27\%), maximum likelihood method (85.83\%) (Foody 2002). If in the past a pixel was very large, being difficult to distinguish entities such as houses, today these images can distinguish cars and even smaller units, such as people who were on the street when acquiring the image (Dey et al. 2010).

Alongside the studies using Landsat images (Herbei et al. 2015), there is a large number of studies using images taken by other satellites. Some of these studies combine object-oriented analysis with the per-pixel analysis in an attempt to achieve results with greater accuracy (AguirreGutierrez, Seijmonsbergen and Duivenvoorden 2012). Another domain that uses quite a lot the image analysis is an attempt to assess plant health and to discover their potential diseases. A large number of studies is devoted also to soil quality. Some of them are using satellite images, but there are also laboratory studies which are using images obtained using spectroscopic sensors in order to identify the changes in the soil after planting (Adar et al. 2014). The methods used for satellites can also be used for airplanes, if the data presents great urgency character. (Natural Resources Canada, 2016).

\section{MATERIALS AND METHODS}

In this paper, we used satellite images LANDSAT (Herbei, 2015) and SPOT, of high spatial 


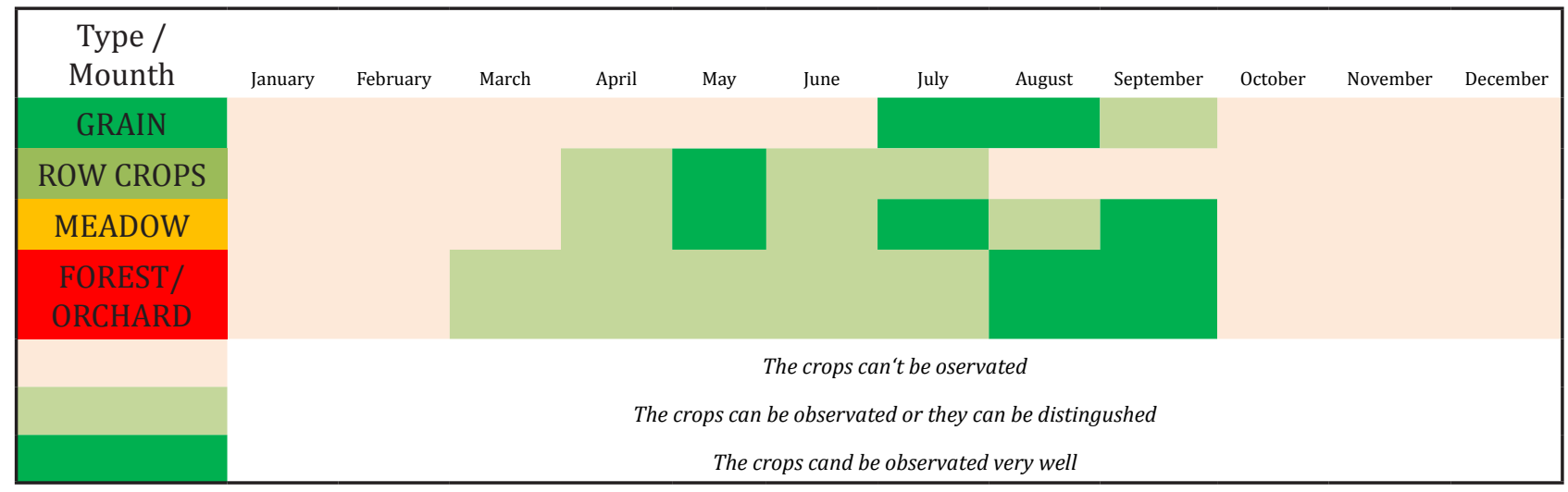

Fig.1. Months when the major crop groups can be seen on satellite images

resolution, with spectral coverage in the visible and infrared field and decent time resolution some images per season, as well as very high resolution images such as WorldView 2 provided by Digital Globe and Quickbird, acquired in 2011, 2013 and 2015.

In order to achieve the classification of agricultural land in the study area (Braila County), taking into account the major crop groups, first it was necessary first to identify them and to determine periods during which they can be identified and the way they will be shown on satellite images. Groups of crops have been developed in line with the methodology and payment categories from the Agency for Payments and Intervention in Agriculture (APIA), namely: straw, row crops, pastures or meadows and forests.

In the straw category there are plants such as: wheat, rye, triticale, barley, two-row barley, etc. In the row crop there are plants such as: corn, sunflower, sugar beet, sorghum, peas, beans, canola, soy, potatoes, etc. (Ministry of Agriculture and Sustainable Development 2013).

For these groups of crops to be identified on satellite images it is important first of all to have the clear time of year when the main phenophases of these types of crops take place in the study area. The months when these cultures can be seen on satellite images appear in the chart below, taking into account the main phenological phases of plants. As can be seen from the diagram in Figure 1 , the images used for the present study, in late April and early July, are great for identifying these groups of crops which are the main focus of the study. Even if a greater number of images, from several months, would have been useful for determining more clearly each of these groups of crops, these two images represent the minimum necessary to enable the identification (De Wit and Clevers 2010).

\section{Unsupervised classification}

Unsupervised classification does not imply preliminary preparation for the analyst regarding terrain features. Pixels are not known at the level of land signification being grouped into clusters based solely on their spectral signature, expressed in images by color tones or shades. (Bogdan M. A., 2007). In our case the resulting image is very uneven color, which is why problems occur in defining classes and especially in defining some subclasses, as we have in this case, agricultural land, with and without vegetation, with grain or row crops. Thus, wrongly attributed significations lead to errors propagated in subsequent analyzes. Although simple at the level of algorithms, unsupervised classification requires experience in selecting the algorithm, establishing the parameters and interpretation of the result. It is useful in the context of using clusters obtained in generating interest areas, in supervised classification.

\section{Supervised classification}

Supervised classification of digital imaging is a classification where classes of objects on the Earth's surface are known in advance on certain limited areas of the image. These areas fall into patterns then developing to rules which can be extended to parts unknown in the image.

In other words, the user identifies several areas on the image that are characteristic of each class of details set. By analyzing the image, each pixel of the image is classified in one of these classes. Thus, supervised classification is based on 

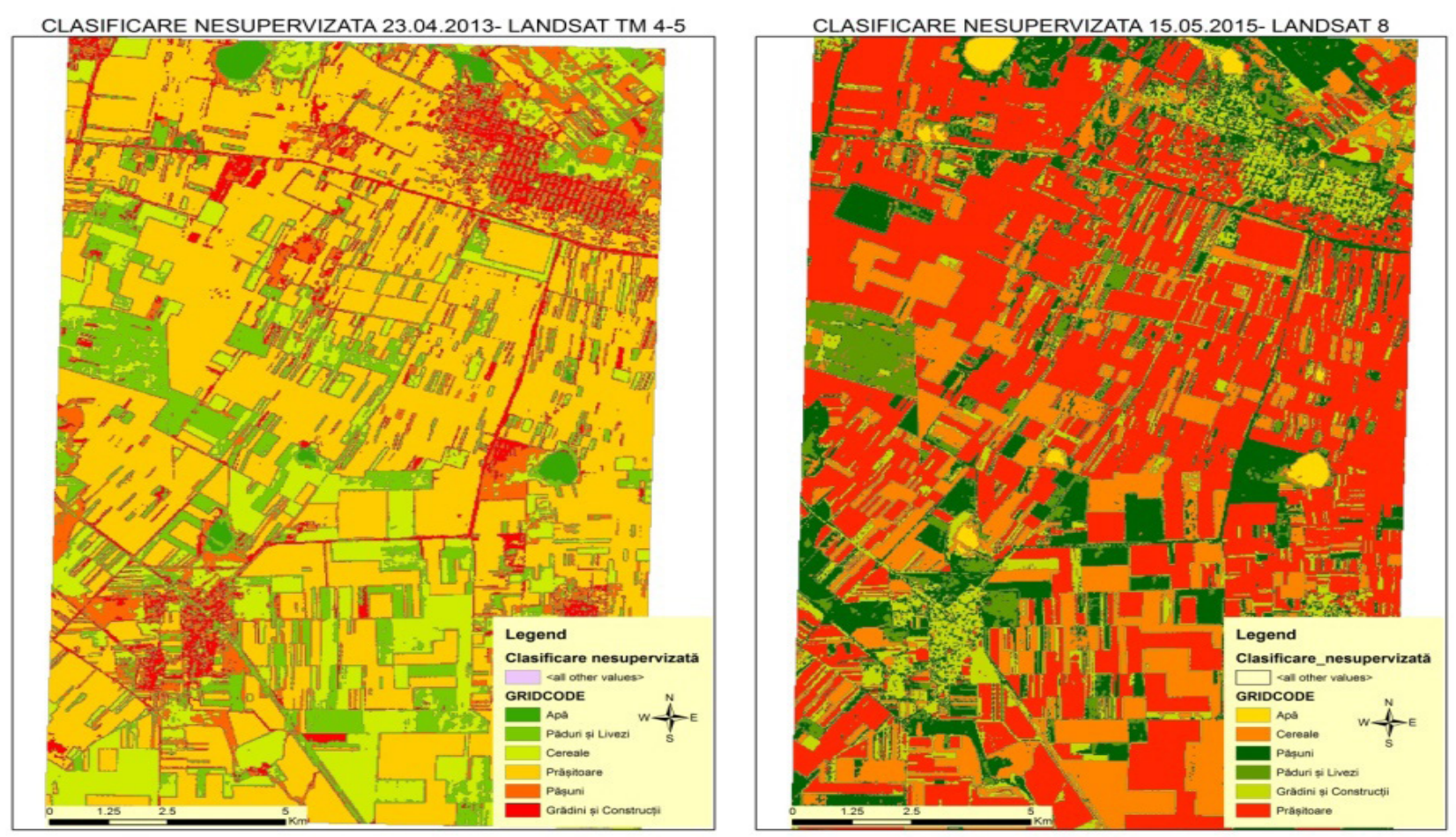

Fig.2. Example of Unsupervised classification of Landsat satellite images

prior knowledge of the surface characteristics of a portion of the image and their use as decision factors in determining the properties of the remaining portions.

Classification of satellite images involved identifying the main classes of crops in the study area. According to the Ministry of Agriculture, the main classes of crops are cereal crops and row crops, but given that the Scheme of Single Surface Payment also includes pastures or hayfields, it was decided to include them, but also the forests in the classification. Once established these four classes of crops, respectively land use, the proper classification was done using unassisted classification algorithms, as well as assisted classification algorithms.

Supervised classification, is a set of mathematical algorithms and statistics, run in digital environment, which is based on tracking each pixel in each spectral band, of a satellite scene or subscene, at the level of digital number and referencing it to a set of sample data created by the analyst. (Bogdan M. A., 2007).

The classification in this case is based on the difference as high as possible regarding the spectral signature from one pixel to another. Since the sample data are a key element and especially the importance of field research and various other sources, we consider this type of classification, the most geographical one.

Supervised classification has three stages:

- preparatory stage, when the analyst identifies sample areas which are delimited and associated with numerical values (eg. forest, pastures, built land, arable land, water etc.)..

- actual classification stage where each pixel in the image is distributed in a class of land cover according to predetermined sample areas, thus pixels that do not find their likeness are reported as unknown.

- final stage, where the new image resulted by classification is processed chromatically, statistically exploited, used in mapping or integrated as a distinct layer in GIS applications.

Preparatory stage, has the highest importance, thus the classification result is based on the accuracy of sample areas, which are groups of pixels of different shapes and sizes, homogenous in terms of geographic significance (eg. forest, pastures, built land, arable land, water, etc.).

Creating these areas sample is handmade, similar to vectorization, around some pixels 


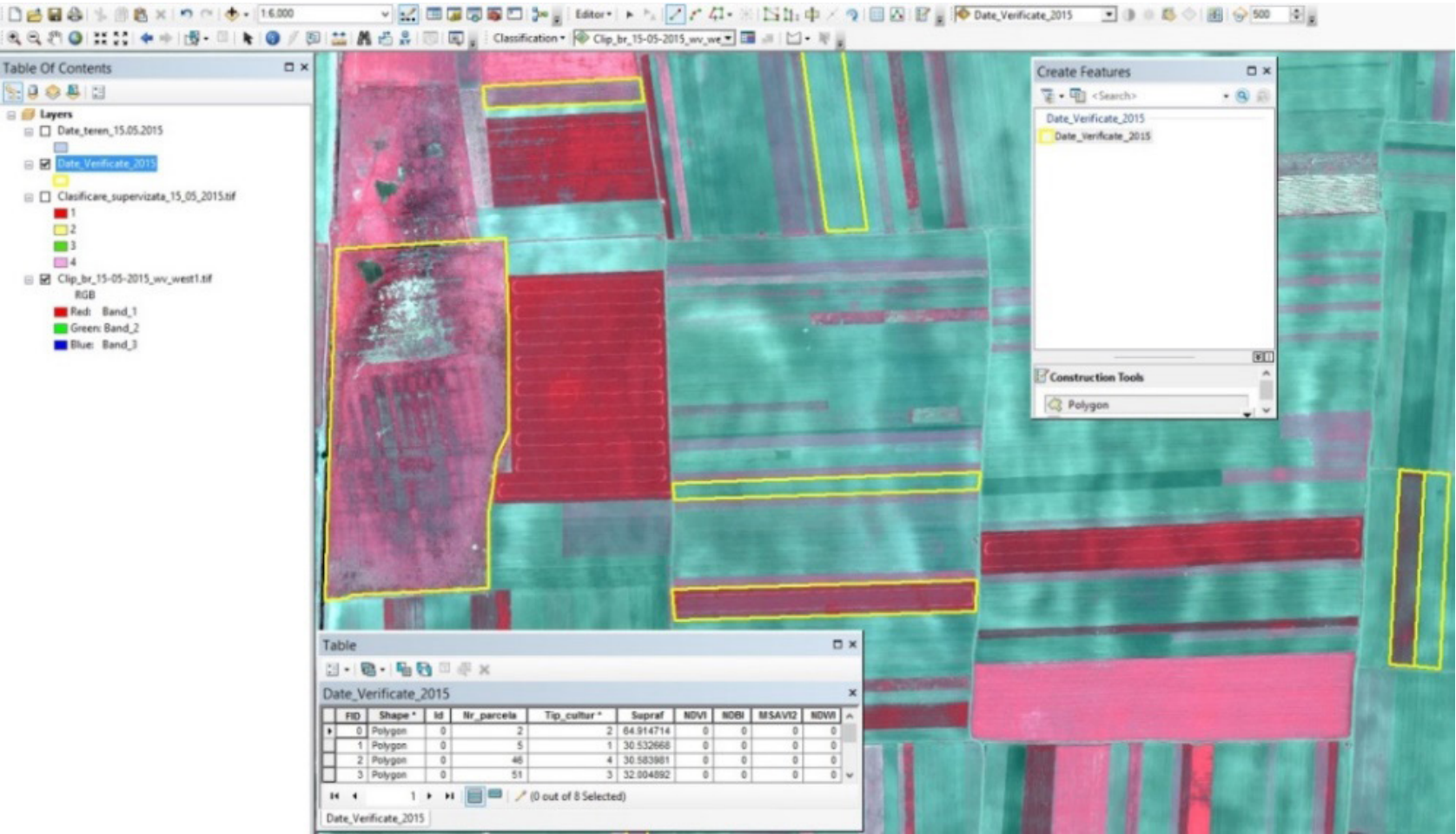

Fig. 3. Sample areas generated on a false color combination resulting from combining reports of shadows elimination.WorldView-2, May 2015

considered as being representative. Classification becomes more effective if elected pixels are more numerous and representative from the spectral and informational point of view. A better space distribution of the image is required, so it can capture the smallest differences at the same class level of the picture, in different lighting conditions. The images were geometrically corrected and rectified to avoid getting large spatial errors.

The stage of actual classification requires

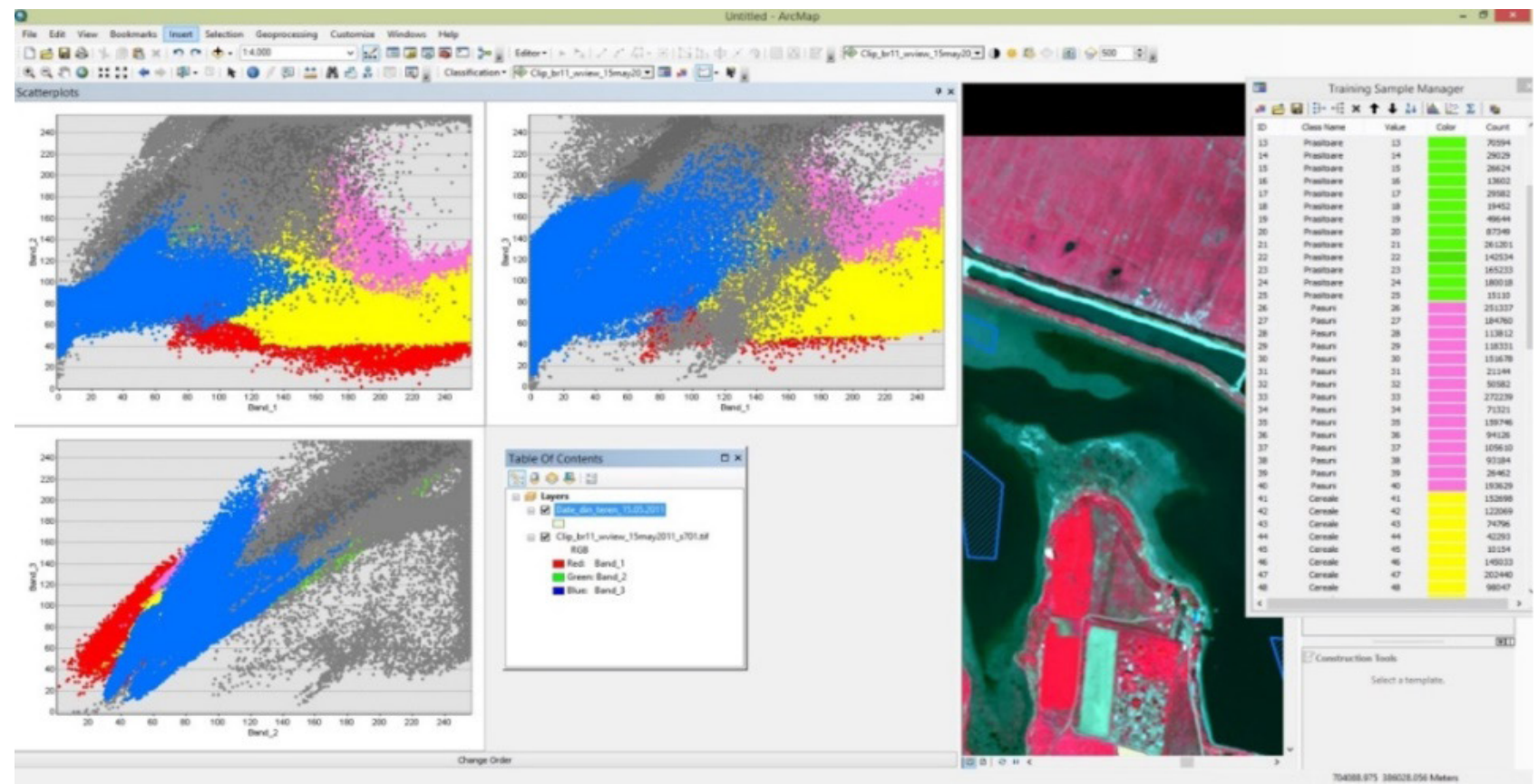

Fig. 4. Gaussian algorithm of Maximum Likelihood by distribution of the samples 
choosing appropriate algorithm by the analyst. The quality of the classification result also depends on this. In this case, the goal is to classify all pixels with smaller errors in relation to the land reality. Running the algorithms require the creation of sample areas, thus there are several types of algorithms:

- Average minimum distance algorithm

- Parallelepiped algorithm

- Maximum Likelihood Gaussian algorithm

- Bayesian algorithm

In this paper we used Gaussian algorithm

of Maximum Likelihood. This algorithm starts from the automatic generation of some Gaussian functions, with bell-shaped graphic that expresses the density of probabilities. Reporting is done in 3D space where the horizontal plane corresponds to system axis to which the digital numbers are reported in the two spectral bands. The vertical axis expresses the probability density of a pixel belonging to one class or another, depending on sample areas.

Distribution of samples collected from satellite images were analyzed using one of three tools on the Image Classification toolbar, respectively Histograms, Scatterplots, Statistics. The algorithm is commonly applied to complex images in terms of spectral signatures. Each pixel is reported to equiprobability functions, regardless of the spectral band. Because of that running requires a longer time, as the image is larger and the number of spectral bands is bigger. Gaussian statistics are generated in all possible combinations of two bands.

Work methodology involved three steps: processing satellite images, satellite images classification, and checking the accuracy of the classification of satellite images. Processing satellite images implies eliminating topographic effects on the satellite images, by rectification using as a reference picture the ortophotoplans on the scale of 1:5000, but also measured land control points.

Classification of satellite images involved identifying the main classes of crops in the study area. According to the Ministry of Agriculture, the main classes of crops are: cereal crops and row crops, but considering that the Scheme of Single Surface Payment also includes pastures or hayfields, it was decided to include them, but also forests, in the classification. Once established these four classes of crops or land use, the proper classification began using both unassisted classification algorithms and assisted classification algorithms.

Because the assisted classification needs exercise areas, the necessary surface was calculated for the study area using an online calculation algorithm of sampling used in statistics (Creative Research Systems), thus for the studied area of 26105 hectares it was required 165 hectares to be used as training areas for a accuracy level of $95 \%$ and an accuracy interval of \pm 10 , as shown in Figure 4.1.

\section{RESULTS AND DISCUSSION}

Supervised classification was performed using ArcGIS software and involved the following steps:

1. Collection of samples;

2. Generating a file containing the spectral signature samples;

\section{Actual Classification.}

For classification we used a scene QuickBird and WorldView - Combination 543 (FALSECOLOR).

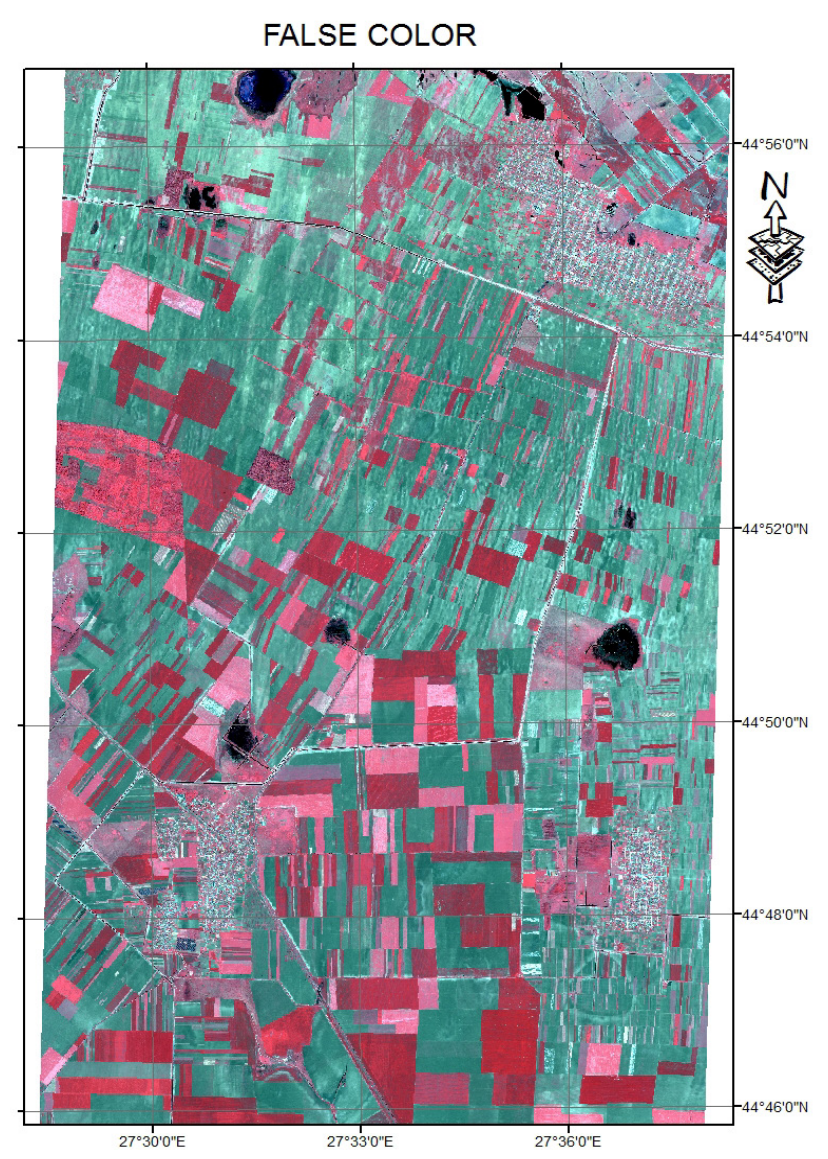




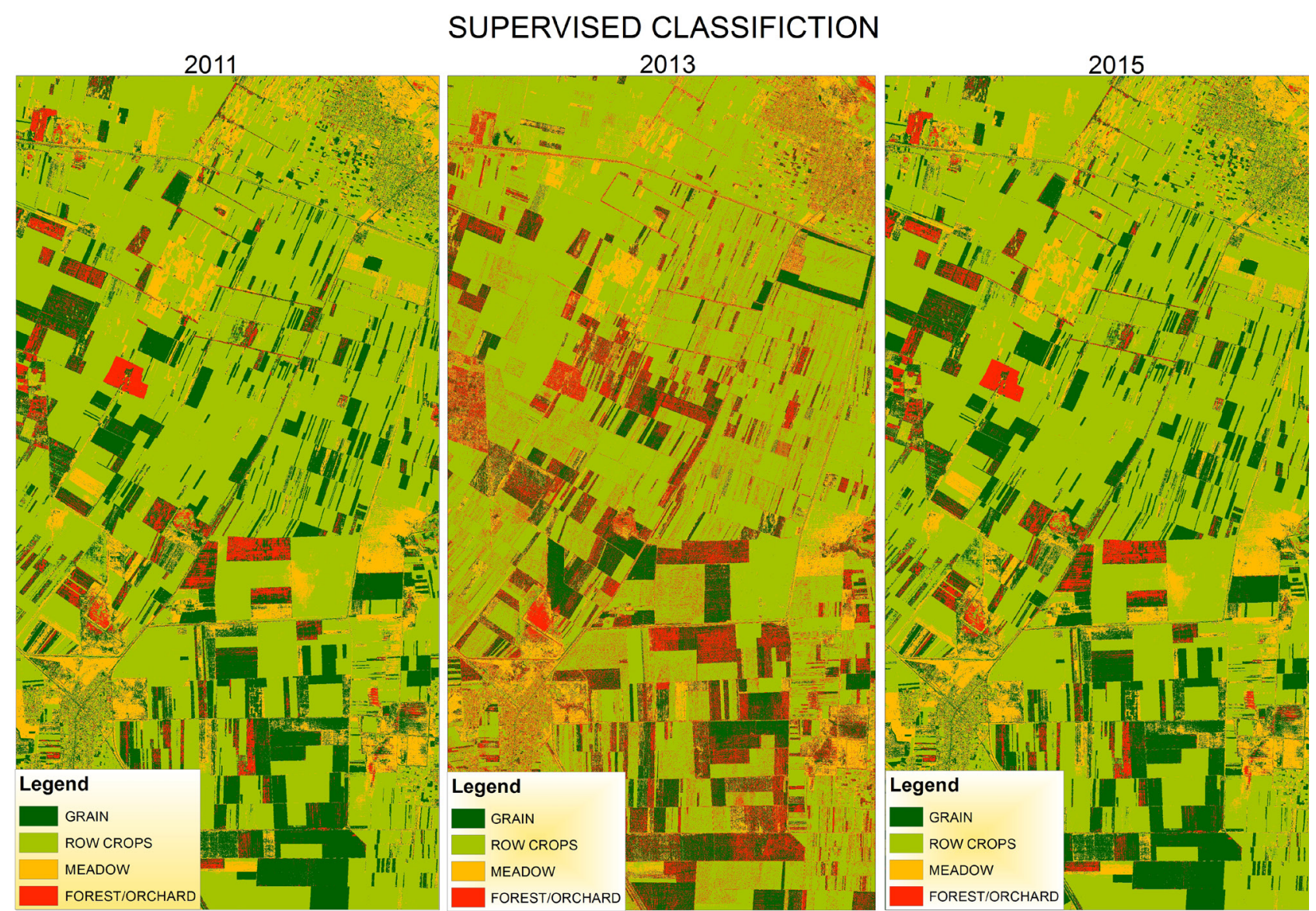

Fig. 5. Studied area - False Color and Supervised Classification Maps 2011 - 2013 - 2015

False Color image was interpreted as follows:

- light pink - meadows and low arable land covered with vegetation

- dark green - bare arable land

- dark blue - minor beds of rivers, lakes cuvettes

- light blue, white - land with rocks, paved roads, concrete constructions etc.

\section{CONCLUSION}

Verifying the accuracy of classification of satellite images was performed using an area equal but different from the training zones used in assisted classification. In order to verify the results we used a method of data validation, very accurate, namely database checked in the field, as the control done by remote sensing of farmers by APIA. This method is accurate, not only because the information is genuine and has a very high accuracy, but it also allows verification of both the classification and segmentation.

Also, this method of validation is more important, because the present study aims to provide an alternative method to the current method, and in the case where the accuracy of this model is similar to the result obtained with the method used at present, the benefits by using it will become categorical because of the much less time and financial resources necessary. Following the classification of very high resolution satellite images, four main classes of crops were successfully identified, some of which are identified even with an accuracy of $99.23 \%$, as the case of row crops in 2011 (Table 1). Evaluation of classification accuracy was determined using a matrix of confusion, which is based on the relation between reference data (ground reality), and the corresponding results of classification. Such matrices are square, the number of rows and columns equal to the number of classes. Accuracy is the measure of the precision of a special classification scheme and it indicates the percentage of a certain class from the land, which 
Tab. 1. Classification accuracy of satellite images

\begin{tabular}{cccccccccc}
\hline & \multicolumn{3}{c}{ Cultivated area(ha) } & \multicolumn{3}{c}{ Classified area(ha) } & \multicolumn{3}{c}{ Classification accuracy (\%) } \\
\hline Crop type & 2011 & 2013 & 2015 & 2011 & 2013 & 2015 & 2011 & 2013 & 2015 \\
\hline Grain & 50,37 & 45,41 & 30,53 & 64,63 & 43,65 & 14,17 & 71,70 & $\mathbf{9 6 , 1 3}$ & 46,42 \\
\hline Row Crops & 51,54 & 56,86 & 64,91 & 51,94 & 54,86 & 56,36 & $\mathbf{9 9 , 2 3}$ & $\mathbf{9 6 , 4 8}$ & 86,82 \\
\hline Meadows & 46,41 & 35,69 & 32,00 & 39,28 & 38,65 & 56,51 & 84,62 & $\mathbf{9 1 , 7 3}$ & 76,56 \\
\hline $\begin{array}{c}\text { Forest/ } \\
\text { Orchard }\end{array}$ & 32,74 & 30,66 & 30,58 & 25,19 & 31,41 & 31,00 & 76,94 & $\mathbf{9 7 , 5 4}$ & $\mathbf{9 8 , 6 5}$ \\
\hline
\end{tabular}

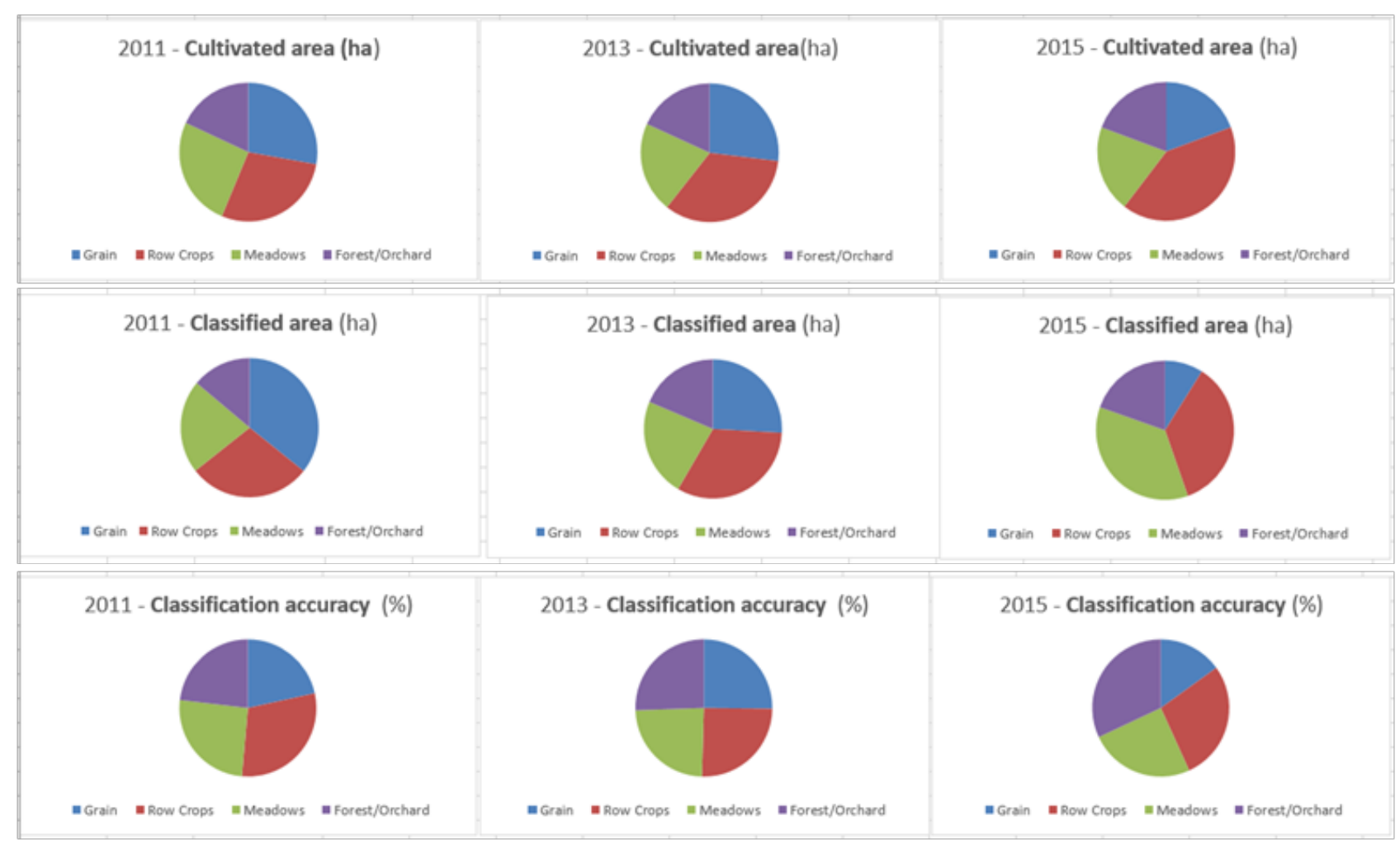

Fig. 6. The graphical representation of the classification results for years 2011 - 2013 - 2015

is classified correctly.

Classification results of satellite image WorldView 2 dated April 13, 2013 are those with the best accuracy of the three images classified, considering that all classes of crops had an accurate classification of over $90 \%$, as can be seen table 4.2. As seen, the accuracy of satellite image classification WorldView 2 from 15.05.2015, is the lowest of the three analyzed regarding classification of grain crops $(46,42 \%)$, according to table 1 and Fig. 5. Given the results of the proposed method in this paper, this appears to be an alternative to consider by the Agency for Payments and Intervention in Agriculture, for the control by remote sensing of the farmers in Romania. The differences resulting from the supervised classification compared to real data from the field are indistinguishable, although the classification results in 2015 were weaker than those of previous years.

\section{REFERENCES}

1. Adar S, Yoel S, Eyal BD (2014). Change detection of soils under small-scale laboratory conditions using imaging spectroscopy sensors.,Geodrma Elsevier Science Inc., 216:19-29.

2. Allan JA (1983).Remote sensing techniques for monitoring and managing irrigated lands. Advances in Space Research, Elsevier Science Inc. 2(8):131-134. 
3. Akgün A, Eronat AH Türk N (2004). Comparing different satellite image clas-sification methods: an application in Ayvalik District, Western Turkey. In: The 4th International Congress for Photogrammetry and Remote Sensing, Istanbul, Turkey.

4. Aguirre-Gutierrez J, Seijmonsbergen A, Duivenvoorden C, Joost F (2012). Optimizing land cover classification accuracy for change detection, a combined pixel-based and object-based approach in a mountainous area in Mexico. Elsevier Science Inc., 34:29-37.

5. Bauer M E (1975). The Role of Remote Sensing in Determining The Distribution and Yield of Crops. Advances in Agronomy, Elsevier Science Inc., 27:271-304.

6. Begov Ungur A (2015). Researches Regarding the Decanting Pond 'Valea Salistei', in View of Areal Rehabilitation and Returning It to the Agricultural Circuit, Journal of Environmental Protection and Ecology, 16(3):1061- 1069.

7. Begov Ungur A (2013). Topographical Survey and Proposals Concerning the Rehabilitation of the Tailings Dump in Gura Rosiei in View of Returning It to the Agricultural Circuit, Journal of Environmental Protection and Ecology, 14(3):1041- 1050.

8. Bogdan MA (2008). Teledetecție., Ed. Credis, București.

9. Bogdan MA (2007). Teledetecție - Introducere în procesarea digitală a imaginilor Vol.1, Ed. Credis, București

10. Coppin P, Nackaerts K, Queen L, Brewer K (2001). Operational monitoring of green biomass change for forest management. Photogrammetric Engineering and Remote Sensing, 67(5): 603-611.

11. De Wit, A.J.W., și J.G.P.W. Clevers. (2010). Efficiency and accuracy of per-field classification for operational crop mapping. International Journal of Remote Sensing (Taylor\&Francis), 25(20):4091-4112.

12. Dey V., Zhang Y., Zhong M. (2010). A Review on Image Segmentation Techniques with Remote Sensing Pespective. ISPRS Commission VII Symposium: 100 Years ISPRS - Advancing Remote Sensing Science, Vienna, Austria, July 5 - 7

13. Foody G.M. (2002). Status of land cover classification accuracy assessment, Remote Sensing of Environment, 80:185-201

14. Herbei M. V., Herbei R. C., Popescu C. A., Bertici R.(2015). Domogled - Valea Cernei National Park monitoring using satellite technology. Ecoterra 12(3):73-78

15. Herbei M, Sala F, Boldea M (2015). Relation of normalized difference vegetation index with some spectral bands of satellite images.

16. Herbei M, (2015). GIS si Modelare cartografica. Universitas, Petroasni, 151-160.

17. Horablaga A, Horablaga M., Grosz D. , Lalescu D., Nita S. , Imbrea I. (2012). The behaviour of the Oenothera biennis L. in the biological culture. Journal of biotechnology, ELSEVIER,

18. Horablaga A, Tabara V, Popescu G, Horablaga N (2013). Results obtained at Oenothera biennis L. Regarding the content and production of oil and determination of linoleic acid, oleic acid in organic production, Research Journal of Agricultural Sciences, 45(3):86-93.

19. Huang S-L., Wang S-H., Bud W.W. (2009). Sprawl in Taipei's peri-urban zone: Responses to spatial planning and implications for adapting global environmental change, Landscape and Urban Planning 90(1-2):20-32.

20. Idso Sherwood B., Ray D. J., Robert J. R. (1977). Remote sensing for agricultural water management and crop yield prediction. Agricultural Water Management, Elsevier Science Inc. 1( 4):299-310.

21. Lu, D. S., Mausel, P., Brondizio, E. S., Moran, E. (2004). Change detection techniques. International Journal of Remote Sensing, 25(12):2365-2407.

22. Meliadis I., Meliadis M. (2011). Multi-temporal Landsat image classification and change analysis of land cover/use in the Prefecture of Thessaloiniki Greece, Proceedings of the International Academy of Ecology and Environmental Sciences 1(1):15-25.

23. Rogan, J., Miller, J., Stow, D., Franklin, J., Levien, L., Fischer, C. (2003). Land cover change mapping in California using classification trees with Landsat TM and ancillary data. Photogrammetric Engineering and Remote Sensing, 69(7):793-804.

24. Rogan, J., Chen, D. (2004). Remote sensing technology for mapping and monitoring land-cover and land-use change. Progress in Planning, 61(4):301-325.

25. Smuleac L., Oncia S., Ienciu A, Bertici R, Smuleac A., Pițiga C. (2013). A study on the possibilities of using groundwater in rural communities in south-western Banat plain. Research journal of agricultural science 45 (2):287-293.

26. Wall, S., Thomas R. W., Brown C.E. (1984). Landsat-based inventory system for agriculture in California. Remote Sensing of Environment, Elsevier Science Inc., 14(13):267-278.

27. William, E. R., William, B. M., Turner, B. L. (1994). Modeling land use and land cover as part of global environmental change. Climatic Change, 28(1-2):45-64.

28. Yuan D, Elvidge CD, Lunetta RS (1998). Survey of multispectral methods for land cover change analysis. In: Lunetta RS, Elvidge CD (Eds.), Remote sensing change detection: Environmental monitoring methods and applications. London: Taylor \& Francis.

29. ***, Natural Resources Canada (2016). Retrieved from http://www.nrcan.gc.ca. 